\title{
Reliability of motor parameters for follow-up after local steroid injection in carpal tunnel syndrome
}

\author{
Salil Gupta, A. K. Tewari ${ }^{1}$, Velu Nair ${ }^{2}$, Aditya Gupta ${ }^{1}$ \\ Departments of Neurology, ${ }^{2}$ Medicine, Armed Forces Medical College, ${ }^{1}$ Department of Neurology, Command Hospital, Pune, \\ Maharashtra, India
}

\begin{abstract}
Background: Local steroid injection is one of the treatment modalities for carpal tunnel syndrome (CTS). Symptomatic and electrophysiological improvement has been previously documented. The electrophysiological parameter, which represents the most consistent change after local steroid injection, is not well-known. Objective: The objective of this study was to evaluate the changes in electrophysiological parameters 1 month after local steroid injection and to determine the parameter that is able to depict improvement across the severity spectrum of CTS. Materials and Methods: Forty-seven patients ( 27 with bilateral disease, 74 hands totally) were included. The electrophysiological parameters studied at baseline included sensory onset latency, sensory nerve action potential amplitude, sensory conduction velocity (CV), distal motor latency, compound muscle action potential amplitude and motor CV. All patients were injected with $40 \mathrm{mg}$ triamcinalone at the wrist. After 1 month, symptomatic improvement from baseline was documented using visual analog score of 10o. Electrophysiology was repeated. Paired $t$ tests were done between baseline electrophysiology parameters and those obtained 1 month after steroid injection for significant improvement. Subgroup analyses were performed in hands with mild to moderate (Grade 3 or less by Bland's classification) and severe disease (Grades 4 and 5). Results: All patients showed a symptomatic improvement. Distal motor latency showed most consistent improvement irrespective of the severity of CTS. In mild to moderate CTS (Grade 3 or less of the classification given by Bland) sensory parameters were recordable and showed significant improvement in addition to distal motor latency. In the subcategory of severe CTS (Grades 4 and 5 of Bland) where sensory parameters are not recordable distal motor latency and the motor CV showed a significant improvement. Conclusion: One month after local steroid injection among the electrophysiological parameters studied distal motor latencies showed most consistent and recordable improvement across the severity spectrum of CTS. This can be used as a single objective parameter to follow-up patients after a local steroid injection to document improvement or relapse. They can also be considered as objective parameter to follow-up patients after surgery.
\end{abstract}

Key words: Carpal tunnel syndrome, electrophysiological parameters, local steroid injection

\section{Introduction}

Carpal tunnel syndrome (CTS) is a common entrapment neuropathy of the median nerve at the wrist. The symptoms include pain, paresthesia and numbness in the hand area supplied by the median nerve. The pain may radiate proximally and may be more at night. Less commonly, patients may develop the motor symptoms such as difficulty in gripping or objects falling out of

\begin{tabular}{|l|l|}
\hline \multicolumn{2}{|c|}{ Access this article online } \\
\hline Quick Response Code: & Website: \\
\hline & www.ruralneuropractice.com \\
\cline { 2 - 2 } & \\
\hline
\end{tabular}

the hand. The treatment of CTS includes non-surgical modalities such as splinting, analgesics, oral steroids, local steroids and for severe disease and surgery. ${ }^{[1]}$ The use of local steroid injections have been mentioned in the literature as early as $1980 .{ }^{[2]}$ A Cochrane review by Marshall et al. in 2007 concluded that local corticosteroid injection for severe CTS provided symptomatic benefit after 1 month compared with placebo. ${ }^{[3]}$ The effect on mild to moderate CTS could not be concluded due to inadequate evidence. Comprehensive guidelines based on available studies provided by the American Academy of Orthopedic Surgeons (2008) suggest the use of local steroid injections prior to considering surgery. ${ }^{[4]}$ These recommendations are based on studies, which have used symptomatic improvement as the primary outcome. Objective electrophysiological parameters showing improvement after local steroid injection has been

Address for correspondence:

Dr. Salil Gupta, Department of Internal Medicine, Armed Forces Medical College, Sholapur Road, Pune - 411 040, Maharashtra, India.

E-mail: chickusalil@yahoo.com 
demonstrated only in very few studies. Some of the short coming of these studies included a small sample size, poorly defined and varied electrophysiological criteria for improvement, exclusion of severe CTS and exclusion of cases with comorbidities. ${ }^{[5-10]}$ There is a need to identify electrophysiological parameter (s), which can be relied upon to document a change and can be used for follow-up after a local steroid injection.

We undertook this study with the following aims:

- To evaluate the changes in the electrophysiological parameters 1 month after local steroid injection in patients with CTS irrespective of the severity

- To identify an electrophysiological parameter that can be consistently relied upon to demonstrate improvement and follow-up patients 1 month after local steroid injection across the severity spectrum of CTS.

\section{Materials and Methods}

All patients referred to the Neurology Out-patient Department of a Tertiary Care Hospital between January 2010 and March 2012 with a presumptive clinical diagnosis of CTS was subjected to electrophysiological studies. Electrophysiology was done by the first author on all patients using a synergy machine. Surface electrodes were used for recording. Due to precautions were taken to keep the hands warm at around $32-33^{\circ} \mathrm{C}$; however, temperature monitoring during the procedure could not be done. Orthodromic technique of stimulation was used to record sensory parameters at the wrist with median nerve being stimulated at second digit and ulnar nerve at the fifth digit. Mid palm stimulation was also done for both the nerves $8 \mathrm{~cm}$ from the recording site at the wrist. Sensory parameters recorded for both nerves included onset latencies, amplitude of sensory nerve action potentials (SNAPs) and conduction velocities (CVs) from both stimulating sites. Motor recording was done using abductor pollicis brevis (APB) for median nerve and abductor digiti minimi for the ulnar nerve. Stimulation was done distally at the wrist and proximally at the elbow. Motor parameters included distal motor latencies, amplitude of compound muscle action potential (CMAPs) and CV. Electrophysiological diagnosis of CTS was made as per the recommendations given by the American Association of Electrodiagnostic Medicine in 2002. ${ }^{[1]]}$ Electrophysiological grading was carried out for all patients as per a scale given by Bland. ${ }^{[12]}$ This study documented the distribution of patients on a scale based upon the nerve conduction study findings, which were independent of the exact normal values. The author demonstrated a highly significant linear relationship between the neurophysiological grading and a numerical score derived from the clinical history. The scale is as follows: Normal (Grade 0); very mild (Grade 1), CTS demonstrable only with most sensitive tests; mild (Grade 2), sensory nerve CV slow on finger/wrist measurement, normal terminal motor latency; moderate (Grade 3), sensory potential preserved with motor slowing, distal motor latency to $\mathrm{APB}<6.5$ ms; severe (Grade 4 ), sensory potentials absent but motor response preserved, distal motor latency to $\mathrm{APB}<6.5 \mathrm{~ms}$; very severe (Grade 5), terminal latency to APB > $6.5 \mathrm{~ms}$; extremely severe (Grade 6), sensory and motor potentials effectively unrecordable (surface motor potential from $\mathrm{APB}<0.2 \mathrm{mV}$ amplitude). ${ }^{[12]}$

All patients diagnosed to have CTS on electrophysiology and who were willing to take a local steroid injection were included in the study. Exclusion criteria were as follows: Previous surgery for CTS, previous local steroid injection, use of oral steroids, Grade 6 (extremely severe) CTS and patients unwilling for local steroid injection. Informed consent was taken from each patient prior to injection. The presence of any comorbidity and symptom (s) at presentation were also documented.

By using the aseptic technique, $1 \mathrm{ml}$ of injection triamcinalone $(40 \mathrm{mg} / \mathrm{ml})$ was injected using a tuberculin syringe and a 26-gauge needle. The site of the injection was at the proximal crease of the wrist just medial to the palmaris longus tendon with the needle angled at $45^{\circ}$ towards the palm and directed slightly medially.

One month after the injection the patients were reviewed. During this period, patients were not permitted to use any other form of therapy like splints or drugs. Patients were first asked to subjectively quantify the degree of improvement (if any) on a visual analog scale (VAS) of 100 on one parameter for which they were most symptomatic prior to the injection. The parameter could include pain, tingling, numbness, nocturnal awakenings or functional improvement. Electrophysiology studies were repeated and the previously mentioned parameters were recorded.

The primary outcome was change in distal motor latency in the median nerve at 1 month after the local injection. The secondary end points were a VAS score of improvement of symptom by at least $50 \%$, change in amplitude of CMAP and motor CV. A VAS score of improvement of symptoms of lesser than $50 \%$ was taken as failure. The motor parameters were preferred over sensory since they were recordable from Grade 1 to Grade 5 CTS (Grade 6 was excluded). CTS is an entrapment neuropathy with features of focal demyelination where out of the motor parameters the distal motor latency is likely to be most affected. Hence, this was chosen as the primary end 
point. Sensory parameters were not taken as they are not recordable in Grade 4 or more CTS. A subgroup analysis was done in mild to moderate CTS (defined as Grade 3 or less) for significant change in sensory onset latency, amplitude of SNAP, sensory CV, distal motor latency, amplitude of CMAP and motor CV. Also in another subgroup consisting of severe and very severe CTS (Grades 4 and 5) the change in distal motor latency, amplitude of CMAP and motor CV were also seen.

Statistical analysis was performed using paired $t$ test to look for a significant difference in the electrophysiological values of each parameter at baseline, which were compared with those at 1 month after a local steroid injection. A $P<0.05$ was considered as significant. SPSS software version 17 was used for analysis.

\section{Results}

Over a 2 year period, 67 patients were screened and 47 patients were selected. 10 patients did not have CTS while four gave previous history of local steroid injection and three of surgery. Three had extremely severe CTS with both SNAPs and CMAP not recordable and hence were excluded. In the study, 27 out of 47 patients selected had bilateral CTS hence 74 hands were included. The baseline characteristics of the patients are given in Table 1 . Nearly, $87 \%$ of our patients were females. Majority (85\%) had Grades 2, 3 or 4 disease and a quarter of our patients had some comorbidity associated. Pain and tingling were the most common symptom at presentation as shown in Table 2.

All patients met the criteria of symptomatic success by reporting a VAS score of symptomatic improvement of at least $50 \%$. 54 hands (73\%) reported $100 \%$ improvement at 1 month in at least one symptom parameter as shown in Table 3.

Mean baseline and follow-up motor parameter in all 74 hands are shown in Table 4. The distal motor latency improved by $9.5 \% 1$ month after local steroid injection and this was found to be highly significant by paired $t$-test. The motor CV and amplitude showed some improvement, but this was not found to be significant.

A subgroup analysis on mild to moderate CTS (Grade 3 or less) in 52 hands showed distal motor latency to improve by $10.3 \%$ from the baseline, which was highly significant by paired $t$-test. However, the motor CV and amplitude showed no significant improvement. All the sensory parameter showed a significant improvement and this is shown in greater detail in Table 5.
A subgroup analysis was also performed in 22 hands with severe (Grade 4) and very severe (Grade 5) CTS as shown in Table 6. The distal motor latency improved by $9.4 \%$ and the motor amplitude improved by $35 \%$, which were found to be significant by paired $t$ test. The motor $\mathrm{CV}$ too showed a $15 \%$ improvement although on paired $t$ test analysis this was not found to be significant [Table 4]. Sensory parameters were not recordable prior to the injection, but after 1 month the injection 10 out of these 22 hands had recordable sensory parameters, however, these have not been analyzed.

\section{Discussion}

After 1 month, a local steroid injection for CTS all our patients irrespective of the severity reported subjective improvement in one parameter for which they were most symptomatic prior to the injection. Our study also

\section{Table 1: Baseline characteristics}

\begin{tabular}{lc}
\hline Characteristic & Number (\%) \\
\hline Females & $41(87.2)$ \\
Bilateral & $27(57.5)$ \\
Right hand involvement & $46 / 74(62.2)$ \\
Mean duration in months (SD) & $41.6(80.5)$ \\
Grade of CTS (total 74 hands) & \\
Grade 1 & $8(10.8)$ \\
Grade 2 & $27(36.5)$ \\
Grade 3 & $17(23)$ \\
Grade 4 & $19(25.7)$ \\
Grade 5 & $3(4.1)$ \\
Comorbidities (total 47 patients) & \\
Diabetes & $6(12.7)$ \\
Hypothyroidism & $3(6.4)$ \\
Rheumatoid arthritis & $2(4.3)$ \\
SLE & $1(2.1)$ \\
\hline CTS: Carpal tunnel syndrome, SD: Standard deviation, SLE: Systemic lupus \\
erythematosis
\end{tabular}

Table 2: Symptom frequency at presentation

\begin{tabular}{lcc}
\hline Symptom at presentation & Frequency $\boldsymbol{n = 7 4}$ & Percentage \\
\hline Pain & 54 & 73.0 \\
Tingling & 44 & 60 \\
Numbness & 32 & 43 \\
Nocturnal awakenings & 14 & 19 \\
Functional compromise & 5 & 7 \\
\hline
\end{tabular}

Table 3: VAS score (out of 100) of symptomatic improvement one month after injection in 74 hands

\begin{tabular}{lcc}
\hline VAS score & Frequency & Percentage \\
\hline 100 & 54 & 73.0 \\
$75-99$ & 18 & 24.2 \\
$50-75$ & 2 & 2.8 \\
$<50$ & 0 & 0 \\
Total & 74 & 100 \\
\hline VAS: Visual analogue scale & &
\end{tabular}


Table 4: Baseline and follow-up median nerve mean motor parameters one month after the injection in 74 hands

\begin{tabular}{|c|c|c|c|c|c|c|}
\hline Parameter of 74 hands & $\begin{array}{l}\text { Baseline } \\
\text { mean (SD) }\end{array}$ & $\begin{array}{c}1 \text { month later } \\
\text { mean (SD) }\end{array}$ & $\begin{array}{l}\text { Difference in the } \\
\text { mean (SD) }\end{array}$ & $\begin{array}{c}\% \text { change } \\
\text { from baseline }\end{array}$ & $\begin{array}{c}95 \% \mathrm{Cl} \text { of the } \\
\text { difference }\end{array}$ & $P$ value \\
\hline Distal motor latency (ms) & $4.3(1.2)$ & $3.9(1.0)$ & $0.4(0.5)$ & 9.5 & $0.3-0.5$ & $<0.01$ \\
\hline Amplitude of CMAP (mV) & $5.5(2.4)$ & $5.7(2.4)$ & $0.2(1.6)$ & 3.6 & $0.1-0.8$ & 0.1 \\
\hline Motor CV $(\mathrm{m} / \mathrm{s})$ & $50.8(11.3)$ & $52.6(10.2)$ & $1.8(10.1)$ & 3.5 & $0.5-4.2$ & 0.1 \\
\hline
\end{tabular}

CMAP: Compound muscle action potential, CV: Conduction velocities, SD: Standard deviation, Cl: Confidence interval

Table 5: Baseline and follow-up median nerve mean motor and sensory parameters one month after the injection in mild CTS (52 hands)

\begin{tabular}{|c|c|c|c|c|c|c|}
\hline Parameters of 52 hands & $\begin{array}{l}\text { Baseline } \\
\text { mean (SD) }\end{array}$ & $\begin{array}{c}1 \text { month later } \\
\text { mean (SD) }\end{array}$ & $\begin{array}{l}\text { Difference in the } \\
\text { mean (SD) }\end{array}$ & $\begin{array}{c}\% \text { change } \\
\text { from baseline }\end{array}$ & $\begin{array}{l}95 \% \mathrm{Cl} \text { of the } \\
\text { difference }\end{array}$ & $P$ value \\
\hline Onset latency sensory (ms) & $3.9(1.0)$ & $3.4(0.8)$ & $0.4(0.3)$ & 10.3 & $0.3-0.5$ & $<0.01$ \\
\hline Amplitude SNAP $(\mu \mathrm{V})$ & 6.8 & 9.7 & $2.9(4.6)$ & 42.6 & $1.6-4.2$ & $<0.01$ \\
\hline Sensory CV (m/s) & $41.2(10.1)$ & $46.4(9.4)$ & $5.2(4.3)$ & 12.6 & $4.0-6.5$ & $<0.01$ \\
\hline Distal motor latency (ms) & $3.9(0.8)$ & $3.5(0.7)$ & $0.4(0.4)$ & 10.3 & $0.2-0.5$ & $<0.01$ \\
\hline Amplitude of CMAP (mV) & $6.3(2.1)$ & $6.6(2.0)$ & $0.4(1.5)$ & 6.3 & $0.1-0.8$ & 0.1 \\
\hline Motor CV (m/s) & $54.9(7.9)$ & $54.8(9.1)$ & $0.1(8.9)$ & 0.2 & $2.4-2.6$ & 0.95 \\
\hline
\end{tabular}

CTS: Carpal tunnel syndrome, SNAP: Sensory nerve action potential, CV: Conduction velocities, CMAP: Compound muscle action potential, SD: Standard deviation, $\mathrm{Cl}$ : Confidence interval

Table 6: Baseline and follow up median nerve mean motor parameters one month after the injection in severe CTS (22 hands)

\begin{tabular}{|c|c|c|c|c|c|c|}
\hline Parameters of 22 hands & $\begin{array}{l}\text { Baseline } \\
\text { mean (SD) }\end{array}$ & $\begin{array}{c}1 \text { month later } \\
\text { mean (SD) }\end{array}$ & $\begin{array}{c}\text { Difference in the } \\
\text { mean (SD) }\end{array}$ & $\begin{array}{c}\% \text { change } \\
\text { from baseline }\end{array}$ & $\begin{array}{c}95 \% \mathrm{Cl} \text { of the } \\
\text { difference }\end{array}$ & $P$ value \\
\hline Distal motor latency (ms) & $5.3(1.2)$ & $4.8(1.1)$ & $0.5(0.8)$ & 9.4 & $0.2-0.9$ & $<0.01$ \\
\hline Amplitude of CMAP (mV) & $3.5(2.0)$ & $4.2(2.2)$ & $0.7(1.7)$ & 35 & $0.1-1.4$ & 0.07 \\
\hline Motor CV $(\mathrm{m} / \mathrm{s})$ & $41.1(12.2)$ & $47.3(11.0)$ & $6.3(11.6)$ & 15.3 & 1.1-11.4 & 0.02 \\
\hline
\end{tabular}

Declaration of $\mathrm{Cl}$. This is to certify that there was no conflict of interest involved in the design, execution or analysis of this study. SD: Standard deviation,

Cl: Confidence interval, CMAP: Compound muscle action potential, CV: Conduction velocities, CTS: Carpal tunnel syndrome

showed that there is a significant improvement in distal motor latency consistently irrespective of the severity of the CTS. We have also demonstrated that in mild to moderate CTS (Grade 3 of Bland) in addition to distal motor latencies there is a significant improvement in all sensory parameters recorded, which include onset latency, amplitude of SNAP and sensory CV. However, amplitude of CMAP and motor CV did not show a significant improvement. In severe to very severe CTS (Grades 4 and 5) in addition to distal motor latencies there is an improvement in motor $\mathrm{CV}$ with the amplitude of CMAP showing a trend toward improvement.

These findings can be explained on the pathophysiological basis of entrapment neuropathy, which is focal demyelination. Early electrophysiological changes (sensory or motor) in focal demyelination are marked by latency prolongation and slowing of CV. Onset of axonal loss is usually marked by the drop in amplitude. In the course of the natural history of CTS sensory parameters are affected earlier and more severely than motor parameters and are soon not recordable. The distal motor latencies too are affected early with prolongation, but stay within the "normal limits." As the severity of the entrapment worsens further prolongation takes place beyond the "normal limits" and focal slowing of motor CV appears. Loss of amplitude of CMAP will take place only in very severe cases once significant axonal loss has started. This was seen in our study too where after a local steroid injection the sensory parameters, although sensitive were not recordable in Grades 4 or 5 CTS and could not be relied upon to document improvement. Among the motor parameters, the distal motor latencies were recordable from Grades 1 to 5 and showed the most consistent improvement. Other motor parameters were not reliable in our study.

So far, following a local steroid injection for CTS, there are no clearly defined electrophysiological parameters for outcome assessment. This stems from the fact that the various studies have used different severity scales for grading CTS, recruited only mild CTS (with intact sensory parameters), defined heterogeneous outcome assessment parameters and various time frames at which assessment was carried out. Giannini et al. found that local injection of triamcinolone $(40 \mathrm{mg})$ in addition to providing symptomatic relief improved distal motor and sensory latencies of the median nerve at the wrist. ${ }^{[5]}$ Agarwal et al. recruited mild CTS defined as distal motor latencies $<7.5 \mathrm{~ms}$ with assessment of multiple parameters, severe CTS and patients with comorbidities were excluded. ${ }^{[10]}$ Hagebeuk and de Weerd 
too had multiple electrophysiological parameters and demonstrated improvement in sensory latencies, SNAP, sensory $\mathrm{CV}$ and CMAP at 6 months, but this was in mild CTS ${ }^{[8]}$ A study on a pregnant woman with CTS by Moghtaderi et al. showed improvement in sensory CV, sensory and motor latency at 3 weeks. ${ }^{[7]}$ Ayhan-Ardiç and Erdem demonstrated improvement in sensory latencies, amplitude, CV and motor distal latencies at 3 weeks and 3 months in 20 hands. ${ }^{[6]}$ The studies by Armstrong et al. ${ }^{[13]}$ Celiker et al., ${ }^{[14]}$ Lucantoni et al. ${ }^{[15]}$ and $\mathrm{O}^{\prime}$ Gradaigh and Merry ${ }^{[16]}$ used electrophysiological parameters only as a secondary outcome.

After 1 month, a local steroid injection symptomatic improvement has been documented earlier and also in our study. ${ }^{[3]}$ However, symptom severity does not necessarily correlate with electrophysiological severity, a relatively symptomatic patient may have a mild disease or a patient with severe CTS on electrophysiology may have mild symptoms. Hence, an objective parameter, which consistently improves after a local steroid injection irrespective of the grade of CTS, is necessary to follow-up patients and also for future studies. We found that distal motor latency is the most consistent electrophysiological parameter showing improvement in all grades of severity of CTS. Sensory parameters although useful in mild to moderate CTS are not recordable in more severe disease and cannot be relied upon for follow-up.

We chose to assess electrophysiological parameters the $2^{\text {nd }}$ time at 1 month because it has been noted earlier that symptomatic response usually starts at 2 weeks. ${ }^{[13]}$ Peak response usually occurs at 1 month.

\section{Conclusion}

Our study has demonstrated that 1 month following a local steroid injection for CTS distal motor latency was the electrophysiological parameter, which showed the most consistent and reliable improvement irrespective of the severity of CTS. This observation can be used to objectively follow-up patients following a steroid injection for the duration of improvement and also document relapse. These findings can also be used to plan future studies using the local steroid injections. They can also be extrapolated to objectively follow-up patients following surgery for CTS. The limitations of our study include lack of controls and follow-up limited to 1 month. Future studies can be planned to overcome these limitations.

\section{References}

1. Assmus H, Antoniadis G, Bischoff C, Haussmann P, Martini AK, Mascharka $Z$, et al. Diagnosis and therapy of carpal tunnel syndrome: Guideline of the German societies of handsurgery, neurosurgery, neurology, orthopaedics, clinical neurophysiology and functional imaging, plastic, reconstructive and aesthetic surgery, and surgery for traumatology. Handchir Mikrochir Plast Chir 2007;39:276-88.

2. van Rossum J, Kamphuisen HA, Wintzen AR. Management in the carpal tunnel syndrome: Clinical and electromyographical follow-up in 62 patients. Clin Neurol Neurosurg 1980;82:169-76.

3. Marshall S, Tardif G, Ashworth N. Local corticosteroid injection for carpal tunnel syndrome. Cochrane Database Syst Rev 2007;2:CD001554.

4. Treatment of Carpal Tunnel Syndrome. Evidence Report. Rosemont (IL): American Academy of Orthopaedic Surgeons; 2008.

5. Giannini F, Passero S, Cioni R, Paradiso C, Battistini N, Giordano N, et al. Electrophysiologic evaluation of local steroid injection in carpal tunnel syndrome. Arch Phys Med Rehabil 1991;72:738-42.

6. Ayhan-Ardiç FF, Erdem HR. Long-term clinical and electrophysiological results of local steroid injection in patients with carpal tunnel syndrome. Funct Neurol 2000;15:157-65.

7. Moghtaderi AR, Moghtaderi N, Loghmani A. Evaluating the effectiveness of local dexamethasone injection in pregnant women with carpal tunnel syndrome. J Res Med Sci 2011;16:687-90.

8. Hagebeuk EE, de Weerd AW. Clinical and electrophysiological follow-up after local steroid injection in the carpal tunnel syndrome. Clin Neurophysiol 2004;115:1464-8.

9. Ayhan-Ardic FF, Erdem HR, Karaoglan B, Yorgancioglu ZR. Short term results of local steroid injection in carpal tunnel syndrome. Turk J Med Sci 1997;27:541-4.

10. Agarwal V, Singh R, Sachdev A, Wiclaff, Shekhar S, Goel D. A prospective study of the long-term efficacy of local methyl prednisolone acetate injection in the management of mild carpal tunnel syndrome. Rheumatology (Oxford) 2005;44:647-50.

11. American Association of Electrodiagnostic Medicine, American Academy of Neurology, and American Academy of Physical Medicine and Rehabilitation. Practice parameter for electrodiagnostic studies in carpal tunnel syndrome: Summary statement. Muscle Nerve 2002;25:918-22.

12. Bland JD. A neurophysiological grading scale for carpal tunnel syndrome. Muscle Nerve 2000;23:1280-3.

13. Armstrong T, Devor W, Borschel L, Contreras R. Intracarpal steroid injection is safe and effective for short-term management of carpal tunnel syndrome. Muscle Nerve 2004;29:82-8.

14. Celiker R, Arslan S, Inanici F. Corticosteroid injection vs. nonsteroidal antiinflammatory drug and splinting in carpal tunnel syndrome. Am J Phys Med Rehabil 2002;81:182-6.

15. Lucantoni C, Grottoli S, Gaetti R. Comparison between he-ne laser therapy and steroid injections in the treatment of idiopathic carpal tunnel syndrome. La Riabilitazione 11992;25:249-56.

16. O'Gradaigh D, Merry P. Corticosteroid injection for the treatment of carpal tunnel syndrome. Ann Rheum Dis 2000;59:918-9.

How to cite this article: Gupta S, Tewari AK, Nair V, Gupta A. Reliability of motor parameters for follow-up after local steroid injection in carpal tunnel syndrome. J Neurosci Rural Pract 2013;4:392-6.

Source of Support: Nil. Conflict of Interest: None declared. 